Délivrance des antipaludiques sur conseil dans les officines privées du district de Bamako, Mali

\title{
Delivery of the antimalaria on advice in pharmacies deprived of the district of Bamako, Mali
}

Diallo $\mathrm{T}^{1 ; 2}$, Denou $\mathrm{A}^{1}$, Coulibaly $\mathrm{BF}^{1}$, Dakouo $\mathrm{B}^{1}$, Tangara $\mathrm{D}^{1,3}$, Coumare $\mathrm{K}^{3}$, Coulibaly $\mathrm{B}^{2 ; 4}$, Loup-Rey $\mathbf{J}^{5}$.

1 Département Sciences du Médicament, Faculté de Pharmacie, Université des Sciences, des Techniques et des Technologies de Bamako, Mali

${ }^{2}$ Centre d'Information Pharmaco-thérapeutique de Bamako, Mali

${ }^{3}$ Laboratoire National de la Santé, Bamako, Mali

${ }^{4}$ Pharmacie de la Côte, Sogoniko, Bamako, Mali

${ }^{5}$ Réseau Médicament et Développement, Marseille, France

Auteur correspondant: Docteur Tidiane Diallo, Maître Assistant en Toxicologie. Département Sciences du Médicament, Faculté de Pharmacie, Université des Sciences, des Techniques et des Technologies de Bamako, Mali. Téléphone : (00223) 631739 30, B.P. : 1805, Email : tidiallo2017@gmail.com

\section{Résumé}

Contexte et objectif : Le paludisme est une maladie bien connue de la population malienne, le conseil à l'officine est de plus en plus demander dans sa prise en charge par la population. En vue de mieux cerner la problématique de délivrance des antipaludiques sans ordonnance, nous avons initié la présente étude avec comme objectif d'étudier la pratique de la délivrance des antipaludiques sans ordonnance conformément aux directives du programme national de lutte contre le paludisme au Mali.

Population et Méthode : Il s'agissait d'une étude transversale descriptive sur la délivrance des antipaludiques sur conseil. Nous avons retenu dans l'étude tous les clients âgés de 15 ans et plus, reçus dans les officines de Bamako pour une demande d'antipaludique sans ordonnance pour lui-même ou pour une autre personne après leur consentement éclairé.

Résultats : L'étude a été réalisée dans 199 sur les 230 officines que compte le district de Bamako, nous avons enregistré 466 demandes de conseil. Le sexe ration M/F était de 1,7 et $28,9 \%$ des clients auxquels le médicament est destiné ont moins 20 ans, dans 52,4\% des cas le client n'est pas le malade. Les combinaisons thérapeutiques à base d'artémisinine ont été les plus conseillés (52\%). La Sulfadoxine Pyriméthamine a été l'antipaludique le plus acheté parmi les antipaludiques non CTA. Dans $21 \%$ des cas les antipaludiques étaient associés aux antibiotiques

Conclusion : L'officine est le premier recours dans le système de santé pour la majorité des clients présentant une suspicion clinique de paludisme.

Mots clés : Conseil, paludisme, pharmacie, Bamako 
Context and Objective: Malaria is a well-known disease of the Malian population; as a result pharmacists are giving more and more advices regarding this disease to the population. In order to better understand the problem of the delivery of antimalarials without prescription, we initiated this study with the objective of studying the practice of dispensing antimalarials without prescription in accordance with the guidelines of Mali's national malaria program.

Patient and Methods: This was a cross-sectional descriptive study on the delivery of antimalarials based on counseling. We included in the study all clients aged 15 years and over who were received in Bamako pharmacies for a non-prescription antimalarial medication for themselves or for another person based on mutual consentment.

Results: The study was carried out in 199 of the 230 pharmacies in the district of Bamako; we recorded 466 requests for advice. The sex ratio $\mathrm{M} / \mathrm{F}$ was 1.7 and $28.9 \%$ of clients for whom the drug was intended were under 20 years of age, in which case $52.4 \%$ of the costumers were not the actual patients. Artemisinin-based combination therapy was the most recommended (52\%). Sulfadoxine Pyrimethamine was the most widely purchased antimalarial among non-CTA antimalarials. In $34.5 \%$ of cases, antimalarials are associated with other therapeutic classes where analgesics / antipyretics constitute $77.6 \%$ of these classes followed by antibiotics $(21 \%)$.

Conclusion: The pharmacy is the first resort in the healthcare system for the majority of clients with clinical suspicion of malaria.

Keywords: Advice, malaria, pharmacy, Bamako

\section{Introduction}

Au Mali, le paludisme demeure l'endémie majeure et la première cause de morbidité et de mortalité dans les groupes les plus vulnérables, à savoir les enfants de moins de cinq ans et les femmes enceintes [1]. Malgré les progrès accomplis dans le domaine de la prévention et du traitement, les statistiques sanitaires nationales révèlent que le paludisme se situe toujours au $1^{\text {er }}$ rang des affections et représente 42 $\%$ des motifs de recours aux soins dans les formations sanitaires dans la population générale [1].

Selon le code de déontologie des pharmaciens français: le conseil à l'officine est l'ensemble des informations mises à la disposition des clients en vue du bon usage des médicaments. Pour cela, le pharmacien et les auxiliaires en pharmacie ont le devoir particulier de conseil lorsqu'il est amené à délivrer un médicament qui ne requiert pas une prescription médicale. Il doit, par des conseils appropriés et dans le domaine de ses compétences, participer au soutien apporté au patient [2].

Les raisons de demande conseil à l'officine sont multiples, allant de la connaissance de ses maux aux nécessités d'économie, en passant par la banalisation du mal. D'un point de vue physiologique, elle peut soulager ou inversement provoquer des effets secondaires divers et d'un point de vue plus comportemental, elle peut induire 
de mauvaises habitudes comme l'interruption des traitements prescrits ou encore l'allongement des délais avant la consultation.

Ces demandes de conseil sont en général précédées d'une automédication, qui se définit comme étant un simple fait de se soigner soi-même. Toutefois, une telle définition sous-entend la présence d'un mal qui justifie la prise en charge, ce qui n'est pas toujours le cas. Dans un sens plus large, l'automédication se rapporte plutôt à une pratique qui vise à prendre soin de sa personne. Elle n'est donc pas seulement la réponse d'un individu à un état de santé problématique mais elle comprend aussi toute action à but préventif et bénéfique pour sa santé [3]. Au Mali, 60\% des malades ou blessés du district de Bamako ont pratiqué des conseils à la pharmacie en 2013. Les raisons financières, confiance aux pharmaciens connaissance du produit ainsi que la non gravité de l'état du patient ont été les principales raisons de demande de conseil à l'officine [1].

La délivrance d'antipaludique sur conseil est très développée dans sa prise en charge. En vue de mieux cerner la problématique liée à la délivrance des antipaludiques sans ordonnance dans le district de Bamako, nous avons initié la présente étude avec comme objectif d'étudier la pratique de la délivrance des antipaludiques sans ordonnance conformément aux directives du programme national de lutte contre le paludisme au Mali.

\section{Population et Méthodes}

Il s'agissait d'une étude transversale descriptive sur la délivrance des antipaludiques sur conseil. Elle s'est déroulée de novembre 2014 à août 2015 dans les officines privées du district de Bamako, capitale du Mali.

La ville de Bamako compte environ $45 \%$ des officines du pays [4]. Elle est traversée d'Ouest en Est par le fleuve Niger et territorialement divisée en six communes (les communes I à IV sur la rive gauche, V et VI sur la rive droite du fleuve) avec une population de 1926748 habitants en 2012 [1]. Chaque commune est divisée en aires de santé et dans chaque aire se trouve plusieurs officines et au moins un centre de santé communautaire (CSCOM) créé et géré par les populations regroupées en association de santé communautaire (ASACO).

Nous avons retenu dans notre étude tous les patients de 15 ans et plus reçus dans les officines du district de Bamako pour une demande de conseil ou un achat d'antipaludique pour lui-même ou pour une autre personne. Dès la validation du questionnaire, nous avons fait quelques tests dans une pharmacie enfin de nous assurer de la compréhension des questions auprès des patients.

Nous avons passé une demi-journée dans toutes les officines pour la réalisation de l'enquête, la taille de l'échantillon des patients n'était pas prédéfinie. La collecte était en fonction de la fréquentation des 
officines. Les pharmaciens et les vendeurs en pharmacie nous envoyaient tous les clients demandant conseils pour le traitement d'une suspicion de paludisme. Une fois face au client nous faisons une petite présentation de l'étude après leur consentement éclairé et volontaire nous procéderons à la phase question réponse dans la langue maternelle.

Dans notre questionnaire nous avons pris en compte les variables suivantes :

- Caractéristiques sociodémographiques des enquêtés

- Analyse du traitement proposé par le pharmacien ou le vendeur en pharmacie

- Compréhension du traitement et comportement des clients face au paludisme.

- Aux sources d'informations des patients sur le traitement et la prévention du paludisme.

Le logiciel statistique Epi-info7 a servi à la saisie et à l'analyse de nos données.

Nous avons obtenu l'autorisation de tous les pharmaciens. Au cours de l'enquête, nous avons donné un code pour chaque officine en vue de garder l'anonymat et la confidentialité des résultats.

\section{Résultats}

Durant notre étude nous avons enregistré 466 cas de demande de conseil dans les officines privées du district de Bamako.
L'étude a été réalisée dans 199 des 230 officines (86,5\% des officines) que compte le district de Bamako (tableau I).

Les hommes constituent $63,3 \%$ des clients et $28,9 \%$ ont au moins 20 ans. Parmi les 138 clientes $3,6 \%$ sont reconnues enceintes. Dans $52,4 \%$ des cas le client n'est pas le malade.

\section{Données concernant le comportement des clients et le traitement acheté.}

Dans 82,8\% les clients ont affirmé venir à la pharmacie pour demande de conseils sur la prise en charge d'une suspicion de paludisme, ils sont $12,8 \%$ à déclarer qu'ils venaient pour achat un antipaludique. Parmi les clients, 29,2\% sont allés à la pharmacie dans les 24 heures qui ont suivi le début des symptômes de la maladie pour acheter des médicaments (tableau II).

Les clients consultent d'autres personnes avant de venir à la pharmacie dans $28,3 \%$ des cas et ils sont $34,3 \%$ à prendre des médicaments avant d'aller en acheter dans une pharmacie. Les clients achètent le total des médicaments dans 93,6\% des cas. Le coût médian de ces médicaments est de $2285^{\text {FCFA }}$, celui des antipaludiques achetés $1400^{\text {FCFA }}$ et celui des CTA 2870 FCFA (tableau III).

Dans 34,5\% des cas les antipaludiques sont associés à d'autres classes thérapeutiques, le antalgiques/antipyrétiques constituent $77,6 \%$ de ces classes. La majeure partie $(89,1 \%)$ des clients connaissaient les 
indications des médicaments conseillés (tableau IV).

\section{Informations générales sur le paludisme}

Une proportion de $33 \%$ des clients ont reçu des informations sur la bonne utilisation des médicaments antipaludiques à travers principalement la télévision et la radio.

Les clients sont $78,8 \%$ à posséder une moustiquaire et $64,8 \%$ à l'utiliser pour se protéger pendant le sommeil. Ces moustiquaires sont imprégnées d'insecticide dans $51,1 \%$ des cas.

Le recours aux médicaments traditionnels est noté chez 38,8\% des clients pour traiter le paludisme et prévenir avec d'autres médicaments. Ils sont $34,1 \%$ à déclarer qu'ils sont soulagés par leurs traitements traditionnels.

\section{Discussion}

La délivrance sur conseil est l'un des modes le plus fréquent d'achat des médicaments dans les pharmacies du district de Bamako (60\%) [1]. En Afrique ce taux est variable selon les pays, nettement plus élevé au Soudan $(81,8 \%)$ [5] et en Égypte $(81,1 \%)$ [6], plus faible en Éthiopie (38,5\%) [7]

Le paludisme étant la pathologie la plus fréquente au Mali, le conseil à l'officine est très fréquent dans les pharmacies. Les demandes de conseil à l'officine s'explique par la confiance portée sur le pharmacien et sur l'antipaludique déjà utilisé, ce constat a été fait au Sénégal par N'Diaye et al [8]. Au Cameroun, ce sont les raisons de coût des consultations et l'éloignement des structures sanitaires qui sont les motifs d'automédication et de demande de conseil selon une étude réalisée par Guillaume 2004 [9]. En République Démocratique du Congo (RDC), en plus des coûts élevés de la consultation médicale et des examens de laboratoires, les prescriptions médicales faites par des professionnels de santé non autorisés, la non vulgarisation de la liste nationale des médicaments essentiels auprès de prestataires, sont autant de facteurs qui favorisent la demande de conseil de la population dans les pharmacies [10].

Les raisons qui amènent un individu à se tourner vers une demande de conseil à l'officine plutôt que vers son médecin ne sont pas seulement liées à la sévérité des symptômes mais peuvent avoir beaucoup d'autres origines. La douleur n'est pas la seule à régler le comportement des gens. On trouve en effet une multitude d'autres facteurs, tant psychologiques que culturels ou économiques qui sont à même d'influencer le comportement des gens visà-vis de leur santé [3]. A ces facteurs peuvent s'ajouter l'accessibilité géographique et financière aux structures de santé

Au Mali dans 52\% des cas de demande de conseil, le client n'est pas le malade. Ces résultats sont similaires [11]. Cette pratique peut s'expliquer par la confiance de ces personnes sur l'antipaludique qu'ils achètent pour le malade. 
Si au Mali, 54\% des patients se rendent à la pharmacie pour une demande de conseil après 48 heures de suspicion du paludisme, au Sénégal et au Kenya, ils étaient $97 \%$ et $91 \%$ à avoir commencer dans les deux jours suivant la survenue des symptômes [12].

La forte prédominance du sexe masculin plus de $60 \%$, a été trouvée dans beaucoup d'étude sur la demande de conseil dans le traitement du paludisme au Mali [11,12]. Par contre c'est le sexe feminin qui prédomine dans une étude réalisé à l'université de Lubumbashi [13]. Ainsi, nous pourront dire que cette pratique dans le traitement du paludisme au Mali est couramment utilisée chez hommes.

Les enfants de moins de 5 ans et les femmes enceintes représentaient respectivement $14 \%$ et $3,6 \%$ de notre échantillon, ceci explique une sous information des parents et des femmes enceinte dans la prise en charge gratuite du paludisme pour ces deux couches des malades du paludisme au Mali [14]. Ce résultat peut s'expliquer par la faible proportion des patients $(33 \%)$ qui ont reçu des informations sur la bonne l'utilisation des médicaments.

Dans $47 \%$ des cas les clients étaient venu payer les médicaments pour leur enfant, ce taux est similaire à ceux de Escourou et al. (41\%) [15].

Les combinaisons thérapeutiques à base d'artémisinine ont été les plus conseillées pour le traitement du paludisme (52\%).
Nos résultats étaient similaires à beaucoup d'autres études réalisées en Afrique subsaharienne : au Gabon (43,8 \%) [16].

La Sulfadoxine Pyriméthamine a été l'antipaludique le plus acheté des antipaludiques non CTA, cela peut être dû à son coût, moins de $500^{\text {FCFA }}$.

Les antalgiques et antipyrétiques (77\%) étaient fortement associés aux antipaludiques, ceci reste logique vu les signes cliniques du paludisme (fièvre, courbature,...). Ils sont utilisés pour faire baisser la température corporelle et diminuer la douleur induites pas le paludisme, en plus ce sont les molécules les plus utilisés au monde.

La présence d'antibiotique (21\%) dans le traitement présomptif du paludisme en conseil met en évidence une thérapie probabiliste entre des infections bactériennes et le paludisme. Une utilisation rationnelle d'antibiotique est obligatoire enfin d'éviter des risques de résistances bactériennes. Des résistances bactériennes ont en effet, été rapportées vis-à-vis des bêta-lactamines et des quinolones, antibiotiques les plus consommés en conseil en RDC [17]. Par ailleurs une étude réalisée sur la Comparaison de la prévalence et de l'évolution de la résistance à l'acide nalidixique et à la ciprofloxacine de Shigella entre l'Europe-Amérique et l'AsieAfrique de 1998 à 2009 a montré une forte résistance [18]. Beaucoup d'autres études ont mis en évidence le risque de la 
résistance microbiologique par la pratique courante de l'auto médicament [19-21].

Dans 19\% des cas les patients ont affirmé avoir pris un médicament avant de venir à la pharmacie. Ceci explique la disponibilité du médicament à la maison, selon Enquête Démographique et de Santé au Mali (20122013), 10\% des automédications par les malades ou blessés ont été effectué à la maison [1].

Ils étaient $94 \%$ des patients à avoir payé la totalité des produits proposés par le pharmacien avec un coût médian de 2285 FCFA, cela met en évidence la confiance que la population porte sur les pharmaciens d'officine. Malgré cette confiance, les pharmaciens d'officine doivent appliquer les recommandations de l'Organisation Mondiale de la Santé sur la prise en charge du paludisme: une confirmation parasitologique rapide par examen microscopique ou par TDR avant de commencer le traitement chez tous les malades pour lesquels il y a suspicion de paludisme [22].

\section{Conclusion}

Il ressort de notre étude dont l'objectif était d'étudier la pratique de la délivrance des antipaludiques sans ordonnance conformément aux directives du programme national de lutte contre le paludisme au Mali, que la délivrance $\mathrm{d}$ 'antipaludique sur conseil est une pratique très fréquente dans les officines de pharmacie du district de Bamako-Mali.
La prise en charge adéquate du paludisme passe obligatoirement par un usage rationnel des médicaments antipaludiques, car une utilisation irrationnelle peut constituer un frein à la lutte contre le paludisme par le développement de la résistance $\mathrm{du}$ parasite et ainsi compromettre l'efficacité des antipaludiques.

L'automédication doit être surveille par les agents de santé en général et en particulier les pharmaciens d'officine par des informations et des sensibilisations des patients sur les risque de l'automédication enfin qu'ils adhèrent aux recommandations de l'OMS pour vaincre le paludisme.

\section{RÉFÉRENCES BIBLIOGRAPHIQUES}

1. Cellule de Planification et de Statistique (CPS/SSDSPF), Institut National de la Statistique (INSTAT/MPATP), INFO-STAT et ICF International, 2014. Enquête Démographique et de Santé au Mali 2012-2013. Rockville, Maryland, USA : CPS, INSTAT, INFO-STAT et ICF International.

2. Code de déontologie des pharmaciens de France. Publié en 1995 : Art. 501548 de la section 1 «Participation à la protection de la santé ».

3. Max Klohn, Isabelle Villommet. L'automédication. Consultable sur l'URL : 
http://www.medecine.unige.ch/enseign ement/apprentissage/module4/immersi on/archives/2007_2008/travaux/08_r_ automedication.pdf

4. Conférence Internationale des Ordres de Pharmaciens Francophones. Mali, données pharmaceutiques. Consultable sur

l'URL :

http://www.ciopf.org/Fiches-des-

pays/Mali

5. Awad AI, Eltayeb BI, Capps PA. Selfmedication practices in Khartoum State, Sudan. Eur J Clin Pharmacol. 2006;62:317-24

6. Sallam SA, Khallafallah NM, Ibrahim NK, Okasha AO. Pharmacoepidemiological study of self-medication in adults attending pharmacies in Alexandria, Egypt. East Mediterr Health J. 2009;15(3):683-91

7. Abay SM, Amelo W. Assessment of self-medication practices among medical, pharmacy, and health science students in gondar university, ethiopia. J Young Pharm. 2010;2(3):306-10

8. Ndiaye P, Tal-Dia A, Diedhiou A, Juergens-Behr A, Lemort JP. Selftreatment of fever in the northern district of Dakar, Senegal. Med Trop. 2006; 66(1):74-8

9. Marie GUILLAUME. Antipaludéens faux ou contrefaits en accès libre au Cameroun. Consultable sur l'URL : https://www.ird.fr/lamediatheque/fiches-d-actualitescientifique/200-antipaludeens-faux- ou-contrefaits-en-acces-libre-aucameroun

10. Ministère de la santé de la République Démocratique du Congo. Rapport narratif: profil pharmaceutique de la République Démocratique du Congo 2011. Juin 2011. Consultable sur l'URL :

http://www.who.int/medicines/areas/c oordination/drc_pharmaceutical_profil e.pdf

11. Sylla H. Contribution à l'analyse de l'automédication avec les antipaludiques au niveau des officines dans le district de Bamako. Thèse pharmacie, Bamako, 2008, 44P

12. Pratique médicale en Afrique francophone/Les pratiques médicales au Sénégal. Consultable sur 1'URL : https://fr.wikibooks.org/wiki/Pratique_ m\%C3\%A9dicale_en_Afrique_franco phone/Les_pratiques_m\%C3\%A9dical es_au_S\%C3\%A9n\%C3\%A9gal

13. Valentin Bashige Chiribagula et al.Prévalence et caractéristiques de l'automédication chez les étudiants de 18 à 35 ans résidant au Campus de la Kasapa de l'Université de Lubumbashi. The Pan African Medical Journal. 2015;21:107.

14. République du Mali, Primature, Secrétariat Général du Gouvernement, Recueil de textes législatifs et Règlementaires sur la santé et le développement social dans le contexte de la décentralisation. Gratuité des 
moyens de prévention et de traitement du paludisme chez l'enfant de moins de cinq ans et chez la femme enceinte dans les établissements de santé. Bamako, 2012 p. 301

15. Escourou B, Bouville B, Bismuth M, Durrieu G, Dustrie S. Automédication des enfants par les parents un vrai risque. Revue du praticien. 2010 ; 60 (6) : 27-34

16. Denise Patricia MawiliMboumba, Marielle Karine BouyouAkotet, Maryvonne Kombila. Usage des antipaludiques en automédication pour le traitement de la fièvre chez les enfants au Gabon. SANTE, vol. 21, $\mathrm{n}^{\circ} 3$, 2011, pages 127-131, 18 réf., ISSN 1157-5999, FRA

17. Muyembe-Tamfum JJ, Veyi J, Kaswa M, Lunguya O, Verhaegen J, Boelaert M. An outbreak of peritonitis caused by multidrug-resistant Salmonella Typhi in Kinshasa, Democratic Republic of Congo. Travel Med Infect Dis. 2009;7(1):40-3.

18. Gu B, Cao Y, Pan S, Zhuang L, Yu R, Peng Z, et al. Comparison of the prevalence and changing resistance to nalidixic acid and ciprofloxacin of Shigella between Europe-America and
Asia-Africa from 1998 to 2009. Int J Antimicrob Agents. 2012;40(1):9-17.

19. Knowledge and practices relating to malaria in a semi-urban area of Cameroon: choices and sources of antimalarials, self-treatment and resistance. Nsagha DS, Njunda AL, Kamga HL, Nsagha SM, Nguedia Assob JC, Wiysonge CS, Tabah EN, Njamnshi AK. Pan Afr Med J. 2011;9:8.

20. Ouédraogo LT, Somé IT, Diarra M, Guissou IP. [Self-medication in the treatment of acute malaria: study based on users of private health drug stores in Ouagadougou, Burkina Faso]. Bull Soc Pathol Exot. 2008 Apr;101(2):124-7.

21. Faye O, Ndir O, Gay O, Bah IB, Dieng $\mathrm{T}$, Dieng $\mathrm{Y}$, Diallo $\mathrm{S}$, DiagneAK. Population practices in the diagnosis of malaria and use of antimalarial drugs in Dakar. Med Trop (Mars). 1995;55(1):47-50

22. Organisation Mondiale de la Santé. Module de formation à la lutte contre le paludisme : Prise en charge du Paludisme, guide du praticien, 2014. Consultable sur l'URL : http://apps.who.int/iris/bitstream/1066 5/112845/3/9789242503975_fre.pdf 
Tableau I : Caractéristiques des malades pour qui les médicaments sont achetés, Bamako

\begin{tabular}{lc}
\hline Caractéristiques & Effectifs (\%) \\
\hline
\end{tabular}

Genre $(n=466)$

Féminin

$138(29,6)$

Masculin

$295(63,3)$

Inconnu

$33(7,1)$

Age $(n=466)$

0 à 5 ans

$65(13,9)$

6 à 19 ans

70(15)

20 ans et plus

$309(66,3)$

Inconnu

$22(4,7)$

Femme enceinte $(\mathbf{n}=138)$

Oui

Non

$49(35,5)$

Inconnu

$84(60,9)$ 
Tableau II : Actions entreprises avant d'acheter des médicaments à la pharmacie, Bamako

\begin{tabular}{lc}
\hline Actions entreprises & Proportion \\
\hline
\end{tabular}

Consultation d'une autre personne avant la pharmacie $(\mathbf{n}=\mathbf{4 6 6}) \quad \mathbf{2 8 , 3}$

Personne consultée $(n=132)$

Médecin

Membre de la famille

Infirmier

Voisin

Autre personne

Sans réponse

11,4

Prise de médicaments avant d'aller à la pharmacie $(n=466)$

34,3

Type de médicaments pris $(n=160)$

Antalgiques/antipyrétiques

Antipaludiques

Antibiotiques

Anti-rhume

Autres médicaments

Ne sait pas

Sans réponse 
Tableau III : Coût des médicaments achetés, Bamako

\begin{tabular}{lccc}
\hline Achat et coût des médicaments & $\begin{array}{c}\text { Proportion } \\
(\mathbf{n = 4 6 6})\end{array}$ & Médiane & $(\mathbf{Q 1} ; \mathbf{Q 3})$ \\
\hline Dépense avant de venir à la pharmacie & 18,9 & $800^{\text {FCFA }}$ & $\left(215^{\text {FCFA }} ; 1345^{\text {FCFA }}\right)$ \\
Le client a acheté le total des médicaments $(\mathrm{n}=436)$ & 93,6 & & \\
Coût des médicaments achetés & & $2285^{\text {FCFA }}$ & $\left(600^{\text {FCFA }} ; 3975^{\text {FCFA }}\right)$ \\
Coût des antipaludiques achetés & & $1400^{\text {FCFA }}$ & $\left(500^{\text {FCFA }} ; 2940^{\text {FCFA }}\right)$ \\
Coût des CTA achetées & & $2870^{\text {FCFA }}$ & $\left(2490^{\text {FCFA }} ; 3395^{\text {FCFA }}\right)$ \\
\hline
\end{tabular}


Tableau IV : Types de médicaments conseillés, Bamako

\begin{tabular}{lc}
\hline Médicaments prescrits & Proportion \\
\hline Antipaludiques (n= 466) & 51,9 \\
CTA & 42,7 \\
Non CTA & 5,4 \\
Autres & $\mathbf{3 4 , 5}$ \\
Autres médicaments associés (n= 161) & \\
& 77,6 \\
Antalgique/Antipyrétiques (Paracétamol) & 21,1 \\
Antibiotiques & 1,2 \\
Antiinflammatoires & 89,1 \\
Le client connaît les indications des médicaments & 14,5 \\
\hline
\end{tabular}

